\title{
In vivo therapeutic success of microRNA-155 antagomir in a mouse model of pulmonary fibrosis induced by bleomycin
}

\author{
Xiaoyuan Sun ${ }^{1,}$, Yu Kang, ${ }^{2,}$, Shan Xue ${ }^{1}$, Jing Zou ${ }^{1}$, Jiabo Xu ${ }^{1}$, Daoqiang Tang ${ }^{3}$, and Hui Qin ${ }^{1}$
}

Departments of ${ }^{1}$ Respiratory, ${ }^{2}$ Cardiology, and ${ }^{3}$ Pathology, Renji Hospital, School of Medicine, Shanghai Jiaotong University, Shanghai, China
Received: March 20, 2019 Revised : August 20, 2019 Accepted: October 7, 2019

\section{Correspondence to}

Hui Qin, M.D.

Department of Respiratory, Renji Hospital, School of Medicine, Shanghai Jiaotong University, No 160, Pujian Road, Shanghai 200127, China

Tel: $+86-68383101$

Fax: +86-2168383101

E-mail: drqinhui@126.com

https://orcid.org/0000-00030538-2952

*'These authors contributed equally to this work.
Background/Aims: MicroRNAs (miRNAs) play critical regulatory roles in the pathogenesis of pulmonary fibrosis. The aim of this study was to explore whether miRNA antagomirs could serve as potential therapeutic agents in interstitial lung diseases.

Methods: A mouse model of pulmonary fibrosis was established by intratracheal injection of bleomycin (BLM). Using microarray analysis, up-regulated miRNAs were identified during the development of pulmonary fibrosis. miR-155 was chosen as the candidate miRNA. Fifteen mice were then randomized into the following three groups: BLM + antagomiR-155 group, treated with BLM plus intravenously injected with antagomiR-155; BLM group, treated with intratracheal BLM plus phosphate-buffered saline (PBS); and a control group, treated with PBS only. Lung tissues were collected for histopathological analysis, hydroxyproline measurement, and Western blotting. Enzyme-linked immunosorbent assays were used for the measurement of cytokines associated with pulmonary fibrosis.

Results: Histological changes and hydroxyproline levels induced by BLM were significantly inhibited by antagomiR-155. The levels of interleukin 4 (IL-4) and transforming growth factor- $\beta$ (TGF- $\beta$ ) expression were increased after BLM treatment. However, miR-155 silencing decreased the expression of IL-4, TGF- $\beta$, and interferon- $\gamma$. TGF- $\beta$-activated kinase $1 /$ mitogen-activated protein kinase kinase kinase $7\left(\mathrm{MAP}_{3} \mathrm{~K}_{7}\right)$-binding protein $2\left(\mathrm{TAB}_{2}\right)$ of the mitogen-activated protein kinase (MAPK) signaling pathway, was activated by BLM and inhibited by in vivo silencing of miR-155 via antagomiR-155.

Conclusions: In vivo treatment with antagomiR-155 alleviated the pathological changes induced by BLM and may be a promising therapeutic strategy for pulmonary fibrosis.

Keywords: Pulmonary fibrosis; MicroRNA-155; Antagomir; Bleomycin

\section{INTRODUCTION}

Interstitial lung diseases are diffuse parenchymal lung disorders associated with lung inflammation and excessive deposition of extracellular matrix, eventually lead- ing to the destruction of lung structure and loss of lung function [1]. Among these diseases, idiopathic pulmonary fibrosis (IPF) is the most lethal and presents with high heterogeneity in its clinical manifestation, with a reported median survival time of approximately 2.8 years [2]. 
MicroRNAs (miRNAs) are a class of small noncoding RNAs, approximately 18 to 22 nucleotides in length [3], that regulate the expression of target genes involved in various physiological processes.

It has been demonstrated that miRNAs play important roles in the pathogenesis of pulmonary diseases [4]. The therapeutic effect of an antagomir targeting $m i R-30 a$, at reducing pulmonary fibrosis, has been validated in vivo [5], indicating that miRNAs may become the next class of therapeutics. However, studies exploring the therapeutic efficacy of single-stranded miRNA inhibitors, known as antagomirs, in IPF are lacking. In this study, microarray analysis was used to investigate the changes in miRNA expression in lung tissue in a mouse model of IPF induced by bleomycin (BLM). miR-155 was identified as an miRNA up-regulated during the development of pulmonary fibrosis. Therefore, we aimed to explore whether in vivo silencing of $m i R-155$, using a synthetic miRNA inhibitor (antagomiR-155), could impede the development of pulmonary fibrosis by targeting a certain signaling pathway.

\section{METHODS}

\section{Mice}

Six-week-old male $\mathrm{C}_{57} \mathrm{BL} / 6 \mathrm{~J}$ (B6) mice were purchased from The Jackson Laboratory (Bar Harbor, ME, USA) and were housed in a pathogen-free environment in the animal facilities of Renji Hospital, affiliated with the Shanghai Jiaotong University School of Medicine (Shanghai, China). All mouse procedures were approved by the Animal Care and Use Committee of Renji Hospital, Shanghai Jiaotong University, School of Medicine (RJ-2018-03-05).

\section{Mouse model of bleomycin-induced pulmonary fibrosis}

Six mice were randomly divided into the following two groups:(1) a BLM group, treated with intratracheal injection of $5 \mathrm{mg} / \mathrm{kg}$ BLM ( $15 \mathrm{mg}$ BLM dissolved in $15 \mathrm{~mL}$ of phosphate-buffered saline [PBS]; Hisun-Pfizer Pharmaceuticals, Shanghai, China) on day 1 [6] and (2) a control group, treated with intratracheal injection of the same volume of PBS on day 1. Mice were sacrificed on day 18 after being anesthetized with an intraperitoneal injec- tion of $3 \%$ chloral hydrate ( $0.01 \mathrm{~mL} / \mathrm{g})$. The left lung lobe was embedded in paraffin for sectioning, while the right lung lobe was microdissected for total RNA extraction and miRNA microarray analysis.

\section{Histopathological analysis}

Lung tissue samples were fixed with a $4 \%$ paraformaldehyde neutral buffer solution for 24 hours, dehydrated in a graded ethanol series, embedded in paraffin, and sectioned at a thickness of 2 to $3 \mu \mathrm{m}$. Paraffin sections were processed with hematoxylin and eosin (H\&E) and Masson's trichrome staining. Lung fibrosis was semi-quantified by systematically scanning stained sections under a microscope using $4 \times$, and 10x objectives. Each successive field was individually assessed for the severity of interstitial fibrosis and was allotted a score between $\mathrm{o}$ (normal lung) and 8 (total coverage of the field with fibrous), using a predetermined Ashcroft scale of severity [7]. After examining the entire section, the mean score of all examined fields was taken as the fibrosis score for the section. X.S. and D.T. assessed the sections and allotted scores independently. Their results were then averaged to determine the final fibrosis score. Both X.S. and D.T. were blinded to the experimental groups.

Total RNA extraction and miRNA microarray analysis Total RNA was isolated from mouse tissues using TRIzol reagent (Invitrogen, Carlsbad, CA, USA), according to the manufacturer's instructions. The quantity and integrity of the extracted RNA were assessed using a Qubit 2.0 instrument (Life Technologies, Carlsbad, CA, USA) and an Agilent 2200 TapeStation (Agilent Technologies, USA), respectively. One microgram of total RNA from each sample was used to prepare RNA libraries using an NEBNext Multiplex Small RNA Library Prep Set for Illumina (New England Biolabs, Ipswich, MA, USA). RNA samples extracted from 3 mice in the same group were pooled and then assayed on a single microarray. The libraries were sequenced on a HiSeq 2500 instrument (Illumina, San Diego, CA, USA) with single-end 5o-bp reads, at Ribobio Co. Ltd. (Guangzhou, China).

\section{Candidate miRNA selection}

BLM induced significant, extensive alveolar wall thickening; massive infiltration of inflammatory cells into the interstitium; and collagen deposition at day 18 
(Supplementary Fig. $1 \mathrm{~B}, \mathrm{1}_{\mathrm{D}},{ }_{1 \mathrm{~F}}$, and $\mathrm{1H}$ ), compared with control group (Supplementary Fig. $1 \mathrm{~A}, 1 \mathrm{C}, 1 \mathrm{E}, 1 \mathrm{G}$ ). The semi-quantitative fibrosis scores for the two groups are presented in Supplementary Fig. 1 I.

Using a single miRNA microarray, 621 miRNAs were found to be expressed above background and 64 miRNAs showed $\mid \log 2$ (fold change) $\mid \geq 1$ and $p<0.05$, after BLM administration, when compared with the control group. Among these miRNAs, 47 showed elevated expression in the BLM group compared with the control group (Supplementary Table 1, Supplementary Fig. 2). The results of hierarchical clustering analysis are shown in Supplementary Fig. 2. miR-155-3p and miR-155-5p showed high levels of expression with $\mid \log 2$ (fold change) $\mid=6.8786$ and 1.6346, respectively $(\mathrm{p}<0.05)$. Based on previous studies, miR-155 was selected for further investigation.

\section{AntagomiR-155 transfection.}

AntagomiR-155 (5'-ACCCCUAUCACAAUUAGCAUUAA-3') was purchased from Ribobio. The Entranster in vivo transfection reagent was obtained from Engreen Biosystem Co. Ltd. (Auckland, New Zealand). Following the manufacturer's instructions, we first prepared reagent A by dissolving $85 \mathrm{nmol}$ of antagomiR-155 in $570 \mu \mathrm{L}$ of autoclaved double-distilled $\mathrm{H}_{2} \mathrm{O}$ and then adding 570 $\mu \mathrm{L}$ of sterile $10 \%$ glucose and mixing well. Next, we prepared reagent B by adding $570 \mu \mathrm{L}$ of sterile $10 \%$ glucose to $570 \mu \mathrm{L}$ of Entranster in vivo transfection reagent and mixing well. Finally, reagents A and B were mixed (1:1) to yield the working solution. Each $330 \mu \mathrm{L}$ aliquot of working solution contained $12 \mathrm{nmol}$ of antagomiR-155.

\section{Study design}

Nineteen 6-week-old male mice were randomly divided into the following three groups: 1) a BLM+antagomiR-155 group $(n=7)$, treated with intratracheal injection of $5 \mathrm{mg} /$ $\mathrm{kg}$ BLM on day 1, plus intravenous injection of antagomiR-155; 2) a BLM group ( $\mathrm{n}=7$ ), treated with intratracheal injection of BLM plus intravenous injection of PBS; and 3) a control group $(n=5)$, treated with intratracheal plus intravenous injection of PBS. Mice in the BLM + antagomiR-155 group received tail vein injections of $330 \mu \mathrm{L}$ of an antagomiR-155 working solution on day 2, 3, 4, 14, 15, and 16 after BLM injection (Fig. 1). After sacrifice on day 18 , serum samples were collected and stored at $-20^{\circ} \mathrm{C}$ for subsequent cytokine measurement. The left lung lobe
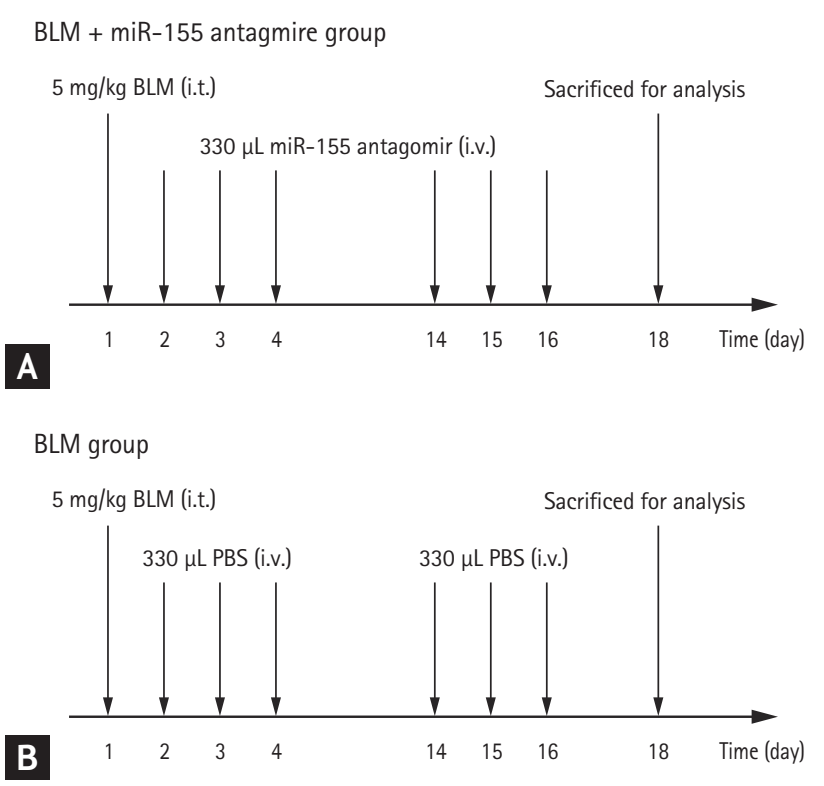

Figure 1. Flowchart of the study. (A) Bleomycin (BLM) + antagomiR-155 group. (B) BLM group. BLM, bleomycin; i.t., intratracheal injection; i.v., intravenous injection; PBS, phosphate-buffered saline.

was embedded in paraffin for further histological analysis and the right lung lobe was microdissected, placed into liquid nitrogen, and stored at $-80^{\circ} \mathrm{C}$ for subsequent polymerase chain reaction (PCR), hydroxyproline (Hyp) measurement, and Western blotting.

\section{Quantitative polymerase chain reaction}

miRNA-155 was quantified in lung tissue by TaqMan quantitative polymerase chain reaction (qPCR), according to the manufacturer's protocol (Life Technologies). U6, a reference small nucleolar RNA, was used as an internal control. The primer sequences used were as follows: 5 '-TTAATGCTAATTGTGATAGGGGT-3' (miRNA-155); 5'-CGCAAATTCGTGAAGCGTTC-3' (U6). The qPCR reactions were performed in a LightCycler 480 Real-Time PCR System (Roche Applied Science, Penzberg, Germany).

\section{Hydroxyproline assessment}

Lung tissue samples (30 to $100 \mathrm{mg}$ wet weight) were lysed in radioimmunoprecipitation assay lysis buffer (JRDUN Bio Co., Ltd., Shanghai, China) at $4^{\circ} \mathrm{C}$ for 30 minutes. The resulting lysates were centrifuged at 3,500 $\times g$ at $4^{\circ} \mathrm{C}$ for 10 minutes and total protein levels in the 
supernatants were quantified using a bicinchoninic acid (BCA) Protein assay kit (Thermo Fisher Scientific, Inc., Waltham, MA, USA). Hyp content in lung tissue extracts was evaluated using a Hyp assay kit (Sengbeijia Bioengineering Institute, Shanghai, China), according to the manufacturer's instructions and results were read on a microplate reader (BioTek Instruments Inc., Winooski, VT, USA) at a wavelength of $450 \mathrm{~nm}$.

\section{Measurement of cytokine levels in serum by ELISA}

The serum concentrations of transforming growth factor- $\beta$ (TGF- $\beta$ ), interferon- $\gamma$ (IFN- $\gamma$ ), and interleukin 4 (IL4) were measured using enzyme-linked immunosorbent assay (ELISA) kits (Sigma, St Louis, MO, USA), according to the manufacturer's instructions.

\section{Western blotting}

The potential targets of miRNA-155 were predicted using miRand, miRWalk 2.0, TargetScan, and miRbase $[8,9]$. Based on these analyses, SMAD2, TGF- $\beta$-activated kinase $1 /$ mitogen-activated protein kinase kinase kinase 7 $\left(\mathrm{MAP}_{3} \mathrm{~K}_{7}\right)$-binding protein 2 ( $\left.\mathrm{TAB}_{2}\right)$, and suppressor of cytokine signaling 1 ( $\mathrm{SOCS} 1$ ) were selected to investigate the molecular signaling pathways underlying pulmonary fibrosis.

Frozen lung tissues were homogenized in RIPA buffer ( $\mathrm{pH}$ 7.6) and total protein concentrations were quantified by BCA assay. Equal amounts of protein (20 to $40 \mu \mathrm{g}$ ) were separated by sodium dodecyl sulfate-polyacrylamide gel electrophoresis (SDS-PAGE) and electrophoretically transferred to a polyvinylidene difluoride membrane (Millipore, Burlington, MA, USA). The membranes were blocked and then incubated with primary antibodies against TAB2 (1:1,000; Proteintech, Rosemont, IL, USA), SOCS1 (1:1,00o; Cell Signaling Technology, Danvers, MA, USA), SMAD2 (1:1,00o; Abways, Shanghai, China). The relative levels of these proteins were determined using image analysis software and were normalized to the levels of $\beta$-actin (1:3,000; Abways). The experiments were repeated three times.

\section{Statistical analysis}

Data analysis was performed using Prism software, version 6.oc (GraphPad, San Diego, CA, USA). Continuous variables are represented as the mean $\pm \mathrm{SE}$ or median. A Student's $t$ test or one-way analysis of variance was

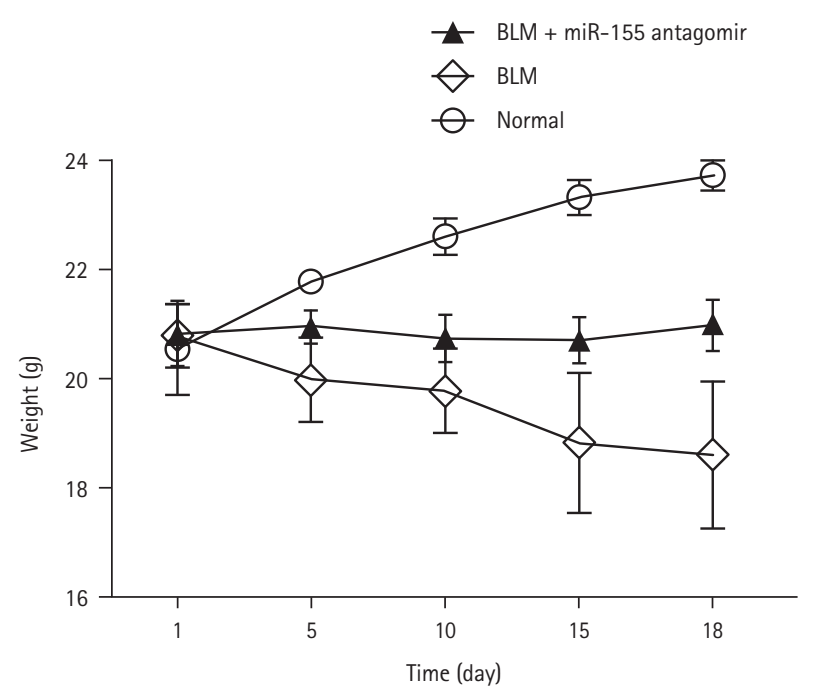

Figure 2. Body weight changes in each group. BLM, bleomycin.

used to determine the differences between mean values for normally distributed variables. The nonparametric Mann-Whitney $U$ test or the Kruskal-Wallis test was used to determine the differences between non-normally distributed variables. A $p$ values less than 0.05 (2-tailed) were considered statistically significant. For microarray data, edgeR was used to compare miRNA levels between the BLM group and the control group. $\mid \log 2$ (fold change) $\mid \geq 1$ and $p<0.05$ were considered statistically significant.

\section{RESULTS}

\section{Mouse survival and body weight}

Two mice from the BLM group and two from the BLM+ antagomiR-155 group died at day 2 (before antagomiR-155 injection) and were excluded from the study. At the end of the study, the body weight of the BLM group decreased from $20.6 \pm 0.4 \mathrm{~g}$ to $19.0 \pm 1.3 \mathrm{~g}$, which was significantly lower than the body weight of the BLM + antagomiR-155 group $(20.5 \pm 0.3 \mathrm{~g}, p=0.01)$ and the control group $(23.7 \pm 0.3 \mathrm{~g}, \mathrm{p}<0.001)$ (Fig. 2).

\section{Attenuated bleomycin-induced pulmonary fibrosis following in vivo silencing of miR-155}

Histopathological analysis showed that compared with control group (Fig. 3A, 3D, $3 \mathrm{G}$, 3J), all BLM-treated mice 

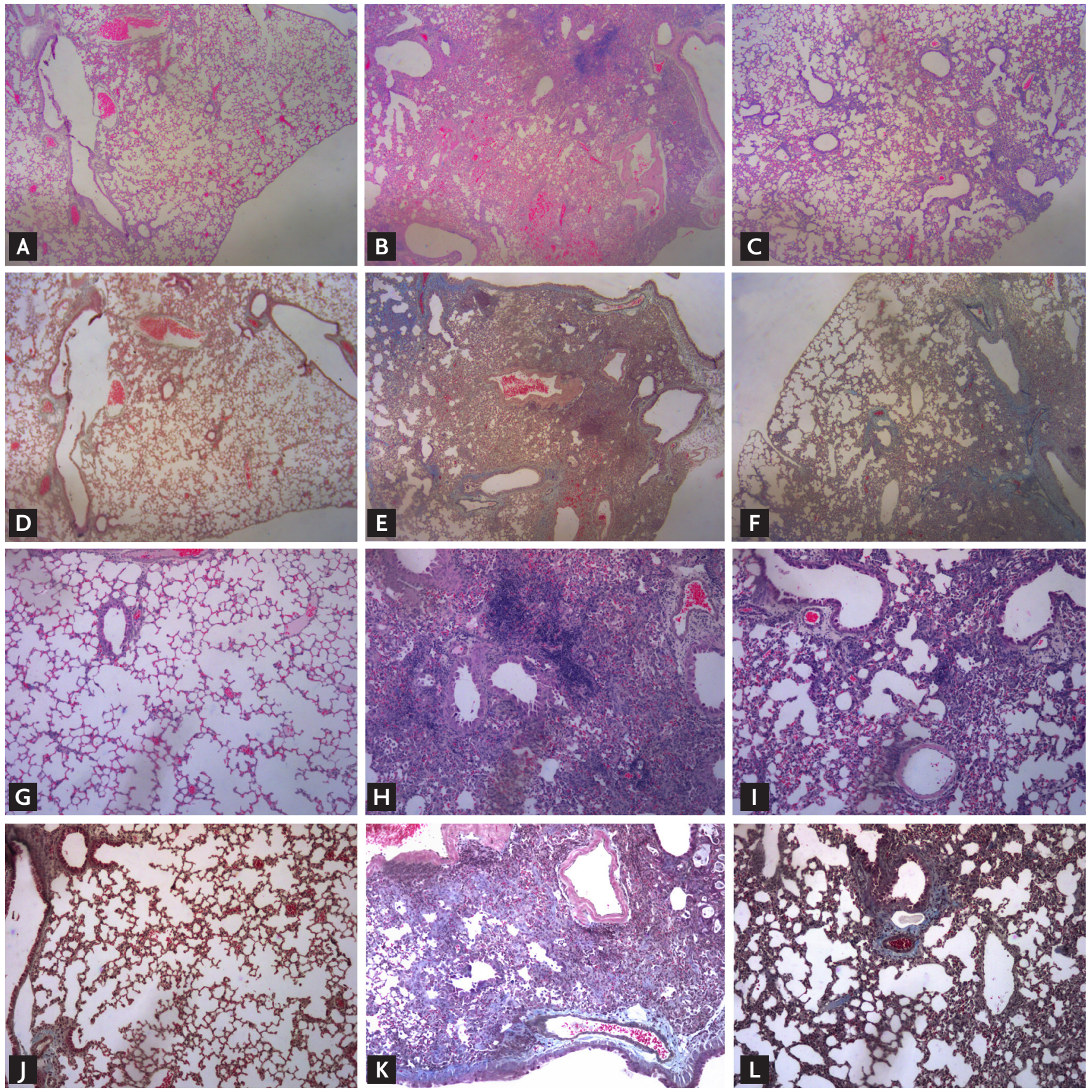

Figure 3. Representative histological lung sections from each group stained with hematoxylin-eosin (A, B, C, G, H, I) and Masson's trichrome stain (D, E, F, J, K. L). Left column: control group; Middle: bleomycin (BLM) group; right: BLM + antagomiR-155 group (A-F: magnification $\times 40, \mathrm{G}-\mathrm{L}$ : magnification $\times 100)$. BLM induced significant alveolar wall thickening, increased inflammatory cell infiltration, and collagen deposition, which were attenuated by antagomiR-155.

developed pulmonary fibrosis (Fig. $3 \mathrm{~B}, 3 \mathrm{E}, 3 \mathrm{H}$, and $3 \mathrm{~K}$ ). However, treatment with antagomiR-155 significantly inhibited the alveolar wall thickening, infiltration of inflammatory cells into the interstitium, and reduction in collagen deposition induced by BLM (Fig. ${ }_{3} \mathrm{C}, 3 \mathrm{~F}, 3 \mathrm{I}$, and
3L). A comparison of Ashcroft scores demonstrated that the degree of pulmonary fibrosis in the BLM group was markedly greater than in the control group, but was impeded by antagomiR-155 treatment (Fig. 4A), indicating that pathomorphological sequelae of BLM-induced pul- 


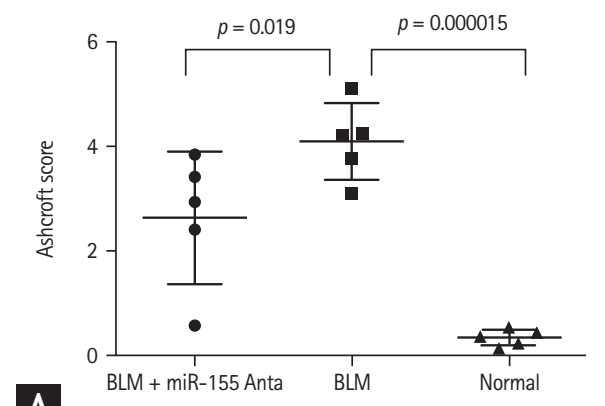

A A

Figure 4. (A) Semi-quantitative analyses of lung tissues for each group using the Ashcroft score. (B) miR-155 expression in lung tissues. AntagomiR-155 greatly reduced $m i R-155$ expression compared with the bleomycin (BLM) group. (C) Hydroxyproline (Hyp) levels in lung tissues of each group. Hyp levels were significantly reduced in the BLM + antagomiR-155 group ( $p<0.001)$.

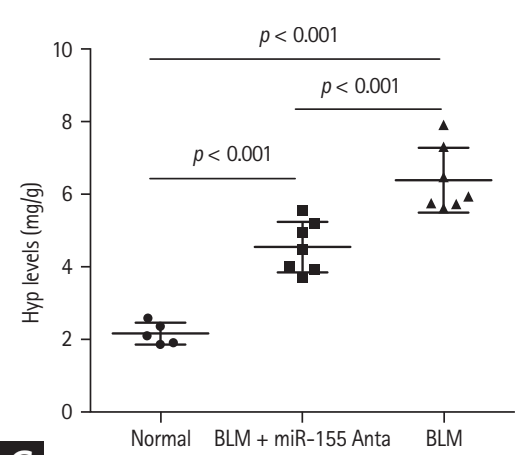

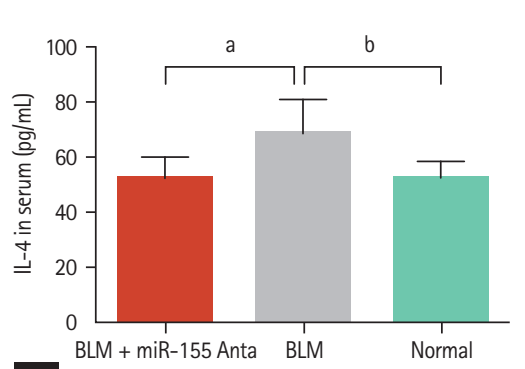

A

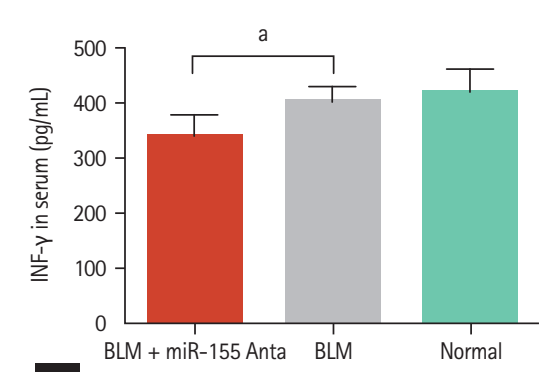

B

Figure 5. Effect of antagomiR-155 on the bleomycin (BLM)-induced production of pro-fibrotic cytokines in serum. (A) Interleukin 4 (IL-4), (B) interferon- $\gamma\left(\right.$ IFN- $\gamma$ ), (C) transforming growth factor- $\beta$ (TGF- $\beta$ ). ${ }^{a} p<0.05$, BLM group vs. BLM + antagomiR-155 group. ${ }^{\mathrm{b}} \mathrm{p}<0.05$, normal group vs. BLM group.

monary fibrosis could be attenuated by in vivo silencing of $m i R-155$ with antagomiR-155.

\section{Downregulated miRNA-155 expression in target tissues}

As shown in Fig. 4B, antagomiR-155 significantly downregulated the expression levels of $m i R-155$ in lung tissues at day 18 .

\section{Hydroxyproline levels}

Hyp content in lung tissues was measured as a representative marker of collagen deposition. As demonstrated in Fig. 4C, Hyp levels were significantly elevated in the BLM group $(p<0.001)$ and this effect was inhibited by antagomiRNA-155 $(p<0.001)$.

\section{Changes in serum cytokine levels}

Serum levels of the profibrogenic cytokines, IL-4 and TGF- $\beta$, were significantly increased in BLM-treated mice compared with control mice $(68.99 \pm 12.03 \mathrm{pg} / \mathrm{mL}$ vs. $52.53 \pm 5.94 \mathrm{pg} / \mathrm{mL}$ and $70.21 \pm 8.78 \mathrm{pg} / \mathrm{mL}$ vs. $56.97 \pm$ $5.13 \mathrm{pg} / \mathrm{mL}$, respectively; $p<0.05$ for both). However, after silencing miRNA-155 in vivo, IL-4, IFN- $\gamma$, and TGF- $\beta$ levels were all decreased compared with their levels in the BLM group $(52.56 \pm 7.57 \mathrm{pg} / \mathrm{mL}$ vs. $68.99 \pm 12.03 \mathrm{pg} /$ $\mathrm{mL}, 355.89 \pm 43.63 \mathrm{pg} / \mathrm{mL}$ vs. $406.24 \pm 25.57 \mathrm{pg} / \mathrm{mL}$, and 57.15 $\pm 6.82 \mathrm{pg} / \mathrm{mL}$ vs. $70.21 \pm 8.78 \mathrm{pg} / \mathrm{mL}$, respectively; $p<0.05$ for all) (Fig. 5).

\section{miRNA-155-associated molecular signaling pathways underly bleomycin-induced pulmonary fibrosis in mice} We assessed the expression levels of proteins involved in various signaling pathways ( $\mathrm{SMAD} 2, \mathrm{TAB} 2, \mathrm{SOCS} 1)$. Among those tested, only TAB2 was found to be activated by BLM and inhibited by miR-155 silencing in vivo (Fig. 6). 

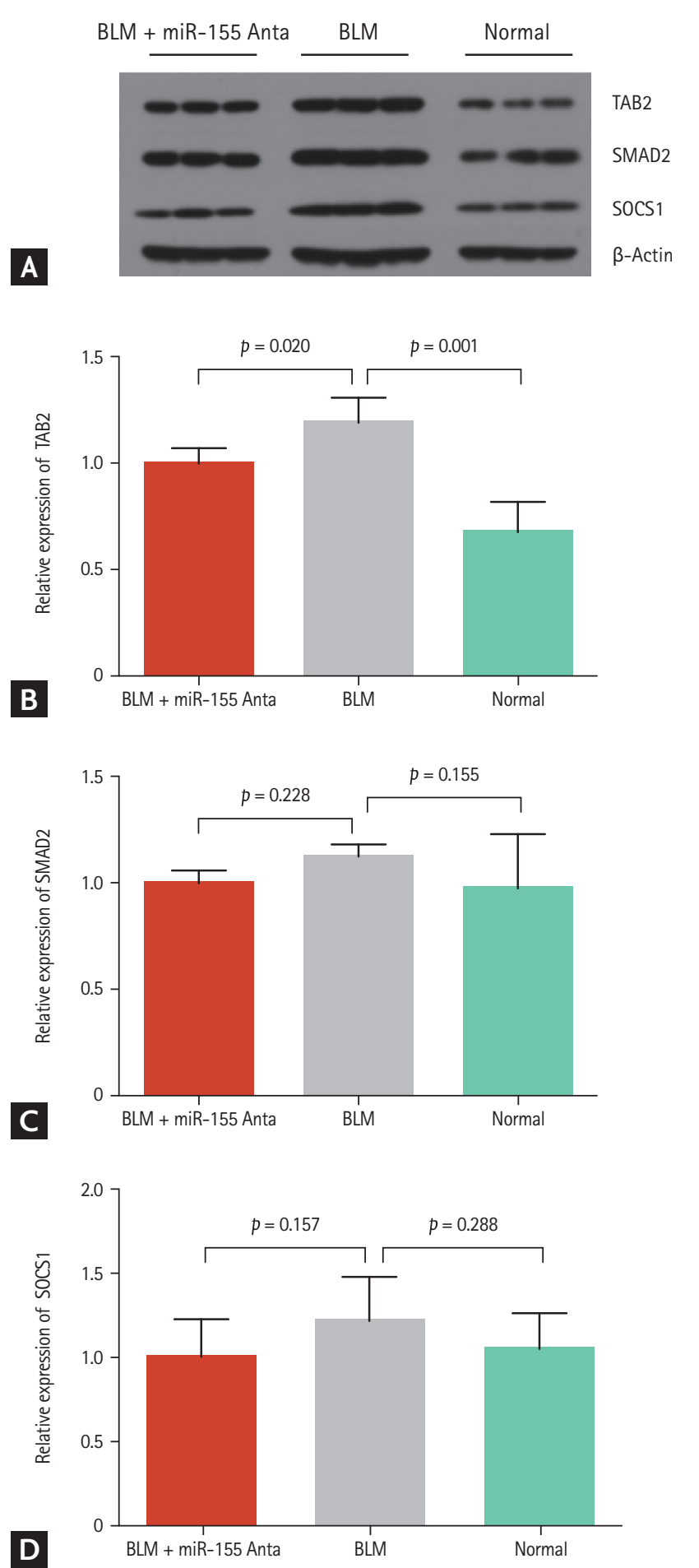

Figure 6. Western blotting analysis. (A) Western blotting analysis of TGF- $\beta$-activated kinase $1 /$ mitogen-activated protein kinase kinase kinase 7 ( $\left.\mathrm{MAP}_{3} \mathrm{~K}_{7}\right)$-binding protein 2 (TAB2), suppressor of cytokine signaling 1 (SOCS1), SMAD2 levels relative to $\beta$-actin in lung tissue from the three groups. (B) TAB2 was activated by bleomycin and was inhibited by in vivo silencing of miR-155 in mice by treatment with antagomiR-155. (C ,D) SMAD2 and SOCS1 showed no different changes between three groups. BLM, bleomycin.

\section{DISCUSSION}

Pulmonary fibrosis is characterized by the presence of fibroblastic foci and myofibroblasts that produce extracellular matrix components, such as collagen type I and III and fibronectin [10]. Intratracheal administration of BLM in B6 mice is the most commonly used model of pulmonary fibrosis and it may also mimic IPF. It has been shown that pulmonary fibrosis develops within 4 weeks after BLM administration, with collagen deposition increasing rapidly after day 10 and peaking on day 21 [6]. However, the development of fibrosis is partially reversible, independent of any intervention [11]. Therefore, in the present study, we assessed the degree of pulmonary fibrosis on day 18. Our analysis showed that lung tissue structural damage, inflammatory cell infiltration, and collagen deposition in the BLM-treated group were similar to what has been reported in a previous study [6].

Studies on the pathogenesis of pulmonary fibrosis have focused on mechanisms regulating the proliferation and activation of myofibroblasts. It is well accepted that microRNAs are important players in the development of fibrosis in multiple organs, including the heart, liver, kidney, and lung $[12,13]$. Using microarray analysis technology, approximately $10 \%$ of assayed microRNAs have been reported to be significantly altered in IPF lungs [4,14]. We demonstrated that miR-155 expression was significantly increased during the development of pulmonary fibrosis. Previously, we found that the progression of acute lung inflammation in lupus is reduced in $m i R-155-/-$ mice and after in vivo silencing of miR-155 [15]. Other studies showed that miR-155 is closely associated with epithelial-mesenchymal interactions [16]. In lung biopsies from patients with IPF, miR-155 is significantly upregulated, in both rapidly progressing and slowly progressing IPF [17]. High expression levels of miR-155 are correlated with the development and the degree of pulmonary fibrosis [12]. These previous results prompted us to select miRNA-155 as a novel target.

Recent clinical efficacy data have underscored the relevance of miRNAs to disease states and the potential for miRNAs to become the next class of therapeutics [18]. Since a single miRNA can regulate numerous target mRNAs within biological pathways, modulation of an miRNA, in principle, allows for the influencing of an entire gene network and the modifying of complex dis- 
ease phenotypes [19]. Antagomirs are synthetic miRNA inhibitors that are considered to be powerful tools for manipulating miRNA levels in vivo. Studies have shown that the injection of antagomirs, in general, is a feasible approach to efficiently down-regulate specific miRNAs in target tissues in a variety of diseases $[20,21]$. In the present study, repeated intravenous injections of antagomiR-155 significantly down-regulated the expression levels of $m i R-155$ in lungs, as assessed by qPCR. This attenuated BLM-induced inflammation and pulmonary fibrosis. To the best of our knowledge, our study is the first to report the effect of antagomir-155 on BLM-induced pulmonary fibrosis in vivo. Therefore, we propose that antagomir injection is a feasible approach to inhibit the expression of distinct miRNAs in target tissues and antagomir-155 may provide a novel therapeutic strategy for pulmonary fibrosis, by inhibiting miRNA-155 in pulmonary tissues.

In view of the molecular pathogenesis of pulmonary fibrosis, we further investigated changes in serum cytokine levels during antagomiR-155 treatment of mice with pulmonary fibrosis. The most important function of $\mathrm{CD}_{4}+\mathrm{T}$ cells is to produce a large quantity of various cytokines, which may contribute to either the inhibition or promotion of fibroblast proliferation, protein synthesis, and collagen production [22]. In the present study, we studied the following 3 cytokines: IL-4, an important initiator in the Th-2-dominated immune response, inducing TGF- $\beta$ production; IFN- $\gamma$, a positive feedback that reinforces the Th1-dominated immune response, inhibiting the production of anti-inflammatory cytokines and promoting the secretion of proinflammatory cytokines; and TGF- $\beta$, an important pro-fibrogenic cytokine. All of these cytokines are essential players in the proliferation and differentiation of fibroblasts, the expression of collagen and fibronectin, tenascin synthesis, and epithelial-mesenchymal transition [23-25]. We demonstrated that, after BLM exposure, IL-4 and TGF- $\beta$ levels increased significantly, while IFN- $\gamma$ levels remained stable. This may be attributed to the small study sample size. However, in the context of clinical IPF, a number of studies of biopsy material have shown that the overall cytokine pattern is more Th2-type than Th1-type [26,27]. Wallace and Howie [26] immunohistochemically examined diffusely infiltrating cells within the interstitium of patients with IPF and showed that most of the diffusely infiltrating mononuclear cells were positive for IL-4 and IL-5 and a small minority of the cells were positive for IFN- $\gamma$. This may partly explain the different cytokine patterns induced by BLM. Recently, emerging evidence has shown that miRNA-155, a typical multifunctional miRNA, is involved in Th cell differentiation, Th1 and Th2 response regulation, and cytokine production $[28,29]$. In our study, we showed that IL-4, IFN- $\gamma$, and TGF- $\beta$ were all reduced by antagomir-155 treatment, which agrees with previous findings that both the Th1-dominated and Th-2 dominated immune responses are suppressed after miR-155 inhibition [30,31]. This may have contributed to the inhibitory effect of antagomir-155 on pulmonary fibrosis.

TGF- $\beta$, which can be induced by IL-4, is a pivotal pro-fibrogenic cytokine. Stimulation of the expression of a number of proinflammatory and fibrogenic cytokines, such as TNF- $\alpha$ and IL- 6 , by TGF- $\beta$-induced signaling, is firmly established as a central mediator of pulmonary fibrosis $[32,33]$. A growing body of evidence shows that TGF- $\beta 1$ activates various SMAD-independent signaling pathways, with or without direct cross-talk with SMAD, and that TGF- $\beta$-activated kinase 1 (TAK1)/MAP $\mathrm{MK}_{7}$ is a major upstream signaling molecule in TGF- $\beta$-induced type I collagen and fibronectin expression [34,35]. The interaction with $\mathrm{TAK}_{1} / \mathrm{MAP}_{3} \mathrm{~K}_{7}$-binding proteins (TABs) is an essential step for TAK1 activation and is necessary for TGF- $\beta$ signal transduction, involved in the activation of inflammatory pathways $[36,37]$. In the present study, BLM led to a significant increase in the levels of TGF- $\beta$ and TAB2, which was in accordance with the aforementioned studies [32,33], thus revealing the role of the TGF- $\beta /$ TAK1-TAB2 signaling pathway in BLM-induced lung fibrosis. This effect could be significantly suppressed by antagomiR-155 treatment. As previously reported, $m i R-155$ has a direct influence on TGF- $\beta$ levels and the knockdown of miR-155 inhibits TGF- $\beta 1$ signaling activation, which may have contributed to the inhibitory effect of antagomiR-155 on pulmonary fibrosis [30,38].

In conclusion, our findings indicated that $m i R-155$ enhanced the inflammatory responses during the development of BLM-induced fibrosis, by regulating multiple inflammatory factors and signaling pathways. We propose that miR-155 antagonists have a potential therapeutic role in pulmonary fibrosis. 


\section{KEY MESSAGE}

1. Interleukin 4 (IL-4) and transforming growth factor- $\beta$ (TGF- $\beta$ ) expression increased and TGF- $\beta$-activated kinase $1 / \mathrm{MAP}_{3} \mathrm{~K}_{7}$-binding protein 2 (TAB2), of the mitogen-activated protein kinase (MAPK) signaling pathway, were activated in a pulmonary fibrosis model.

2. Intravenous injection of antagomiR-155 reduced miRNA-155 levels in lung tissue.

3. AntagomiR-155 alleviated the pathological changes of pulmonary fibrosis.

4. AntagomiR-155 inhibited IL-4, TGF- $\beta$, and interferon $-\gamma$ and $\mathrm{TAB} 2$ expression.

\section{Conflict of interest}

No potential conflict of interest relevant to this article was reported.

\section{Acknowledgments}

This work was supported by the Shanghai Natural Science Foundation (16ZR1420600) and the Research Program of Shanghai College Teachers (101005.26.23).

\section{REFERENCES}

1. Antoniou KM, Margaritopoulos GA, Tomassetti S, Bonella F, Costabel U, Poletti V. Interstitial lung disease. Eur Respir Rev 2014;23:40-54.

2. Tzilas V, Koti A, Papandrinopoulou D, Tsoukalas G. Prognostic factors in idiopathic pulmonary fibrosis. Am J Med Sci 2009;338:481-485.

3. Filipowicz W. RNAi: the nuts and bolts of the RISC machine. Cell 2005;122:17-20.

4. Pandit KV, Milosevic J, Kaminski N. MicroRNAs in idiopathic pulmonary fibrosis. Transl Res 2011;157:191-199.

5. Zhang S, Liu H, Liu Y, et al. miR-3oa as potential therapeutics by targeting TET1 through regulation of Drp-1 promoter hydroxymethylation in idiopathic pulmonary fibrosis. Int J Mol Sci 2017;18:E633.

6. Liu W, Wan J, Han JZ, et al. Antiflammin-1 attenuates bleomycin-induced pulmonary fibrosis in mice. Respir Res 2013;14:101.

7. Ashcroft T, Simpson JM, Timbrell V. Simple method of estimating severity of pulmonary fibrosis on a numerical scale. J Clin Pathol 1988;41:467-470.

8. Chang JT, Nevins JR. GATHER: a systems approach to interpreting genomic signatures. Bioinformatics 2006;22:2926-2933.

9. Kanehisa M, Goto S, Furumichi M, Tanabe M, Hirakawa M. KEGG for representation and analysis of molecular networks involving diseases and drugs. Nucleic Acids Res 2010;38(Database issue):D355-D360.

10. Wynn TA. Common and unique mechanisms regulate fibrosis in various fibroproliferative diseases. J Clin Invest 2007;117:524-529.

11. Moeller A, Ask K, Warburton D, Gauldie J, Kolb M. The bleomycin animal model: a useful tool to investigate treatment options for idiopathic pulmonary fibrosis? Int J Biochem Cell Biol 2008;40:362-382.

12. Kato M, Putta S, Wang M, et al. TGF-beta activates Akt kinase through a microRNA-dependent amplifying circuit targeting PTEN. Nat Cell Biol 2009;11:881-889.

13. Ji J, Zhang J, Huang G, Qian J, Wang X, Mei S. Over-expressed microRNA-27a and $27 \mathrm{~b}$ influence fat accumulation and cell proliferation during rat hepatic stellate cell activation. FEBS Lett 2009;583:759-766.

14. 14. Xie T, Liang J, Guo R, Liu N, Noble PW, Jiang D. Comprehensive microRNA analysis in bleomycin-induced pulmonary fibrosis identifies multiple sites of molecular regulation. Physiol Genomics 2011;43:479-487.

15. Zhou S, Wang Y, Meng Y, et al. In vivo therapeutic success of microRNA-155 antagomir in a mouse model of lupus alveolar hemorrhage. Arthritis Rheumatol 2016;68:953964.

16. Pottier N, Maurin T, Chevalier B, et al. Identification of keratinocyte growth factor as a target of microRNA-155 in lung fibroblasts: implication in epithelial-mesenchymal interactions. PLoS One 2009;4:e6718.

17. Oak SR, Murray L, Herath A, et al. A micro RNA processing defect in rapidly progressing idiopathic pulmonary fibrosis. PLoS One 2011;6:e21253.

18. Janssen HL, Reesink HW, Lawitz EJ, et al. Treatment of HCV infection by targeting microRNA. N Engl J Med 2013;368:1685-1694.

19. van Rooij E, Olson EN. MicroRNA therapeutics for cardiovascular disease: opportunities and obstacles. Nat Rev Drug Discov 2012;11:860-872.

20. Lin CW, Chang YL, Chang YC, et al. MicroRNA-135b promotes lung cancer metastasis by regulating multiple 
targets in the Hippo pathway and LZTS1. Nat Commun 2013;4:1877.

21. Brock M, Samillan VJ, Trenkmann M, et al. AntagomiR directed against miR-20a restores functional BMPR2 signalling and prevents vascular remodelling in hypoxia-induced pulmonary hypertension. Eur Heart J 2014;35:32033211.

22. Jakubzick C, Kunkel SL, Puri RK, Hogaboam CM. Therapeutic targeting of IL-4- and IL-13-responsive cells in pulmonary fibrosis. Immunol Res 2004;30:339-349.

23. Gharaee-Kermani M, Denholm EM, Phan SH. Costimulation of fibroblast collagen and transforming growth factor betal gene expression by monocyte chemoattractant protein-1 via specific receptors. J Biol Chem 1996;271:17779-17784.

24. Makhluf HA, Stepniakowska J, Hoffman S, Smith E, LeRoy EC, Trojanowska M. IL-4 upregulates tenascin synthesis in scleroderma and healthy skin fibroblasts. J Invest Dermatol 1996;107:856-859.

25. Gein SV, Sharavieva IL. Effect of rotation and immobilization stress on IL-1 $\beta$, IL-2, IL-4, and IFN- $\gamma$ production by splenocytes under opiate receptor blockade in vivo. Dokl Biol Sci 2014;454:69-71.

26. Wallace WA, Howie SE. Immunoreactive interleukin 4 and interferon-gamma expression by type II alveolar epithelial cells in interstitial lung disease. J Pathol 1999;187:475-480.

27. Lukacs NW, Hogaboam C, Chensue SW, Blease K, Kunkel SL. Type $1 /$ type 2 cytokine paradigm and the progression of pulmonary fibrosis. Chest 2001;120:5S-8S.

28. Zhang YY, Zhong M, Zhang MY, Lv K. Expression and clinical significance of miR-155 in peripheral blood $\mathrm{CD}_{4}(+) ; \mathrm{T}$ cells of patients with allergic asthma. Xi Bao Yu Fen Zi Mian Yi Xue Za Zhi 2012;28:540-543.

29. Sabri A, Grant AV, Cosker K, et al. Association study of genes controlling IL-12-dependent IFN- $\gamma$ immunity: $\mathrm{STAT}_{4}$ alleles increase risk of pulmonary tuberculosis in
Morocco. J Infect Dis 2014;210:611-618.

30. Xi W, Zhao X, Wu M, Jia W, Li H. Lack of microRNA-155 ameliorates renal fibrosis by targeting PDE3A/TGF- $\beta 1 /$ Smad signaling in mice with obstructive nephropathy. Cell Biol Int 2018;42:1523-1532.

31. Malmhall C, Alawieh S, Lu Y, et al. MicroRNA-155 is essential for $\mathrm{T}(\mathrm{H}) 2$-mediated allergen-induced eosinophilic inflammation in the lung. J Allergy Clin Immunol 2014;133:1429-1438.

32. Fernandez IE, Eickelberg O. The impact of TGF- $\beta$ on lung fibrosis: from targeting to biomarkers. Proc Am Thorac Soc 2012;9:111-116.

33. Zhang D, Liu B, Cao B, et al. Synergistic protection of Schizandrin B and Glycyrrhizic acid against bleomycin-induced pulmonary fibrosis by inhibiting TGF- $\beta 1 /$ Smad2 pathways and overexpression of NOX4. Int Immunopharmacol 2017;48:67-75.

34. Ono K, Ohtomo T, Ninomiya-Tsuji J, Tsuchiya M. A dominant negative TAKı inhibits cellular fibrotic responses induced by TGF-beta. Biochem Biophys Res Commun 2003;307:332-337.

35. Kim SI, Kwak JH, Zachariah M, He Y, Wang L, Choi ME. TGF-beta-activated kinase 1 and TAK1-binding protein 1 cooperate to mediate TGF-beta1-induced MKK3-p38 MAPK activation and stimulation of type I collagen. Am J Physiol Renal Physiol 2007;292:F1471-F1478.

36. Xu C, Ren G, Cao G, et al. miR-155 regulates immune modulatory properties of mesenchymal stem cells by targeting TAK1-binding protein 2. J Biol Chem 2013;288:11074-11079.

37. Zhu S, Pan W, Song X, et al. The microRNA miR-23b suppresses IL-17-associated autoimmune inflammation by targeting TAB2, TAB 3 and IKK- $\alpha$. Nat Med 2012;18:10771086.

38. Zhang D, Cui Y, Li B, Luo X, Li B, Tang Y. miR-155 regulates high glucose-induced cardiac fibrosis via the TGF- $\beta$ signaling pathway. Mol Biosyst 2016;13:215-224. 
Supplementary Table 1. 64 microRNA (miRNA) expression considered significant in miRNAs array

\begin{tabular}{|c|c|c|c|}
\hline miRNA ID & Up/down & Log2 (fold change) & $p$ value \\
\hline mmu-miR-3962 & Down & -4.7985 & $2.66 \mathrm{E}-07$ \\
\hline mmu-miR-26a-1-3p & Down & -6.6486 & $3.69 \mathrm{E}-\mathrm{O} 5$ \\
\hline mmu-miR-676-3p & Down & -1.4751 & 0.000333276 \\
\hline mmu-miR-351-5p & Down & -1.2299 & 0.000919868 \\
\hline mmu-miR-486a-5p & Down & -1.1037 & 0.001853524 \\
\hline mmu-miR-486b-5p & Down & -1.1037 & 0.001855498 \\
\hline mmu-let-7d-3p & Down & -1.2476 & 0.00295935 \\
\hline mmu-miR- 6538 & Down & -6.5648 & 0.004969991 \\
\hline mmu-miR-615-3p & Down & -6.3219 & 0.007013723 \\
\hline mmu-miR-5122 & Down & -6.2225 & 0.008304311 \\
\hline mmu-miR-29c-3p & Down & -1.1487 & 0.008436648 \\
\hline mmu-miR-3097-5p & Down & -6.1225 & 0.010325417 \\
\hline mmu-miR-3086-5p & Down & $-5 \cdot 9619$ & 0.010905805 \\
\hline mmu-miR-503-3p & Down & -1.0463 & 0.011217259 \\
\hline mmu-miR-8120 & Down & -5.9069 & 0.011612619 \\
\hline mmu-miR-505-5p & Down & $-5 \cdot 79$ & 0.013595984 \\
\hline mmu-miR-1298-5p & Down & -1.1126 & 0.016513841 \\
\hline mmu-miR-147-5p & Up & 9.8765 & $1.10 \mathrm{E}-\mathrm{o} 8$ \\
\hline mmu-miR-38o-3p & Up & 8.1615 & $5.68 \mathrm{E}-08$ \\
\hline mmu-miR-217-5p & Up & 7.86 & 1.95 E-०6 \\
\hline mmu-miR-7b-5p & Up & 2.936 & $2.28 \mathrm{E}-\mathrm{o} 6$ \\
\hline mmu-miR-21a-5p & Up & 2.4333 & $6.56 \mathrm{E}-06$ \\
\hline mmu-miR-677-5p & Up & 8.5622 & $1.03 \mathrm{E}-05$ \\
\hline mmu-miR-147-3p & Up & 2.5974 & 0.00011343 \\
\hline mmu-miR-122-5p & Up & 3.03 & 0.000122172 \\
\hline mmu-miR-146b-5p & Up & 2.1576 & 0.000219567 \\
\hline mmu-miR-881-3p & Up & 7.7923 & 0.000357116 \\
\hline mmu-miR-199a-5p & Up & 2.0854 & 0.000401226 \\
\hline mmu-miR-21a-3p & Up & 2.7254 & 0.000413955 \\
\hline mmu-miR-19b-3p & Up & 3.3536 & 0.000576011 \\
\hline mmu-miR-132-3p & Up & 1.9691 & 0.000655514 \\
\hline mmu-miR-335-3p & Up & 1.9765 & 0.000907502 \\
\hline mmu-miR-146b-3p & Up & 1.7995 & 0.001346736 \\
\hline mmu-miR-493-5p & Up & 6.7638 & 0.00223029 \\
\hline mmu-miR-212-5p & $\mathrm{Up}$ & 2.019 & 0.002759906 \\
\hline mmu-miR-184-3p & Up & 1.868 & 0.003005214 \\
\hline mmu-miR-1945 & Up & 6.4207 & 0.003958909 \\
\hline mmu-miR-19a-3p & Up & 2.6147 & 0.004468483 \\
\hline mmu-miR-155-3p & Up & 6.8786 & 0.004825971 \\
\hline mmu-miR-212-3p & Up & 2.0242 & 0.005300568 \\
\hline
\end{tabular}




\section{Supplementary Table 1. Continued}

\begin{tabular}{|c|c|c|c|}
\hline miRNA ID & Up/down & Log2 (fold change) & $p$ value \\
\hline mmu-miR-466i-3p & $\mathrm{Up}$ & 6.0804 & 0.006724962 \\
\hline mmu-miR-377-3p & Up & 6.7234 & 0.007484173 \\
\hline mmu-miR-6540-3p & Up & 6.0224 & 0.007778091 \\
\hline mmu-miR-6539 & Up & 6.5899 & 0.008034547 \\
\hline mmu-miR-145a-5p & Up & 1.8567 & 0.008982992 \\
\hline mmu-miR-214-3p & Up & 1.3876 & 0.009024278 \\
\hline mmu-miR-488-3p & Up & 6.4919 & 0.009081962 \\
\hline mmu-miR-1930-5p & Up & 5.9148 & 0.009166195 \\
\hline mmu-miR-218-1-3p & Up & 5.9773 & 0.00922696 \\
\hline mmu-miR-3084-3p & Up & 5.9148 & 0.009371423 \\
\hline mmu-miR-335-5p & Up & 1.6403 & 0.012168791 \\
\hline mmu-miR-342-3p & Up & 1.4216 & 0.01252695 \\
\hline mmu-miR-494-3p & Up & 2.3083 & 0.013097159 \\
\hline mmu-miR-7a-5p & Up & 1.9291 & 0.015247416 \\
\hline mmu-miR-298-5p & Up & 1.5537 & 0.016350824 \\
\hline mmu-miR-155-5p & Up & 1.6346 & 0.017597784 \\
\hline mmu-miR-511-5p & Up & $3 \cdot 5081$ & 0.027910279 \\
\hline mmu-miR-132-5p & Up & 1.2599 & 0.030190829 \\
\hline mmu-miR-96-5p & Up & 1.3662 & 0.032459014 \\
\hline mmu-miR-148a-5p & Up & 1.1117 & 0.038260701 \\
\hline mmu-miR-223-3p & Up & 1.4601 & 0.042584605 \\
\hline mmu-miR-152-5p & Up & 1.2243 & 0.042721888 \\
\hline mmu-miR-29b-1-5p & Up & 6.6629 & 0.044129757 \\
\hline mmu-miR-21c & Up & 1.5384 & 0.047778651 \\
\hline
\end{tabular}




\section{KJIM}
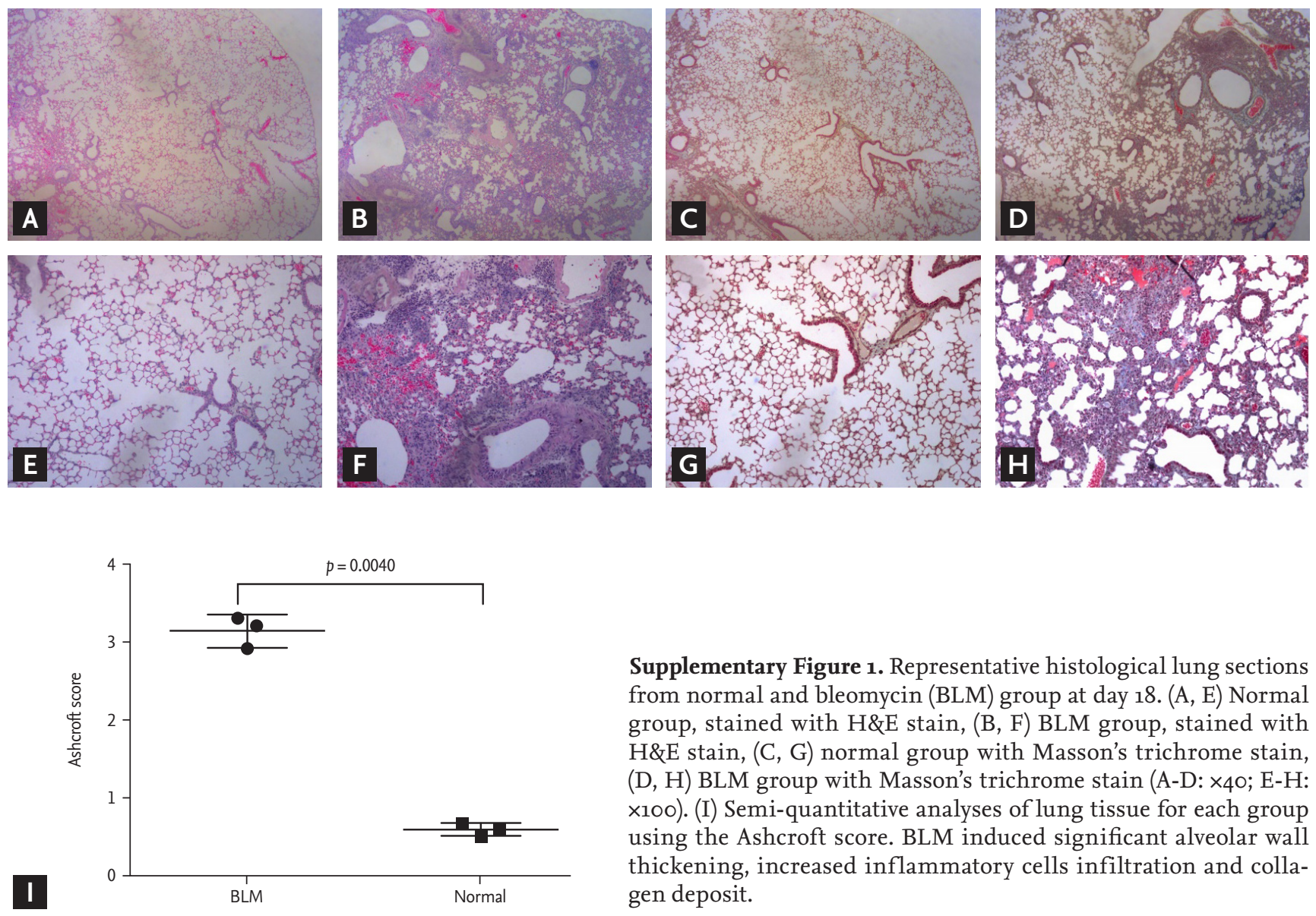

Supplementary Figure 1. Representative histological lung sections from normal and bleomycin (BLM) group at day 18. (A, E) Normal group, stained with H\&E stain, (B, F) BLM group, stained with H\&E stain, (C, G) normal group with Masson's trichrome stain, (D, H) BLM group with Masson's trichrome stain (A-D: $\times 40 ; \mathrm{E}-\mathrm{H}$ : $\times 100)$. (I) Semi-quantitative analyses of lung tissue for each group using the Ashcroft score. BLM induced significant alveolar wall thickening, increased inflammatory cells infiltration and collagen deposit. 


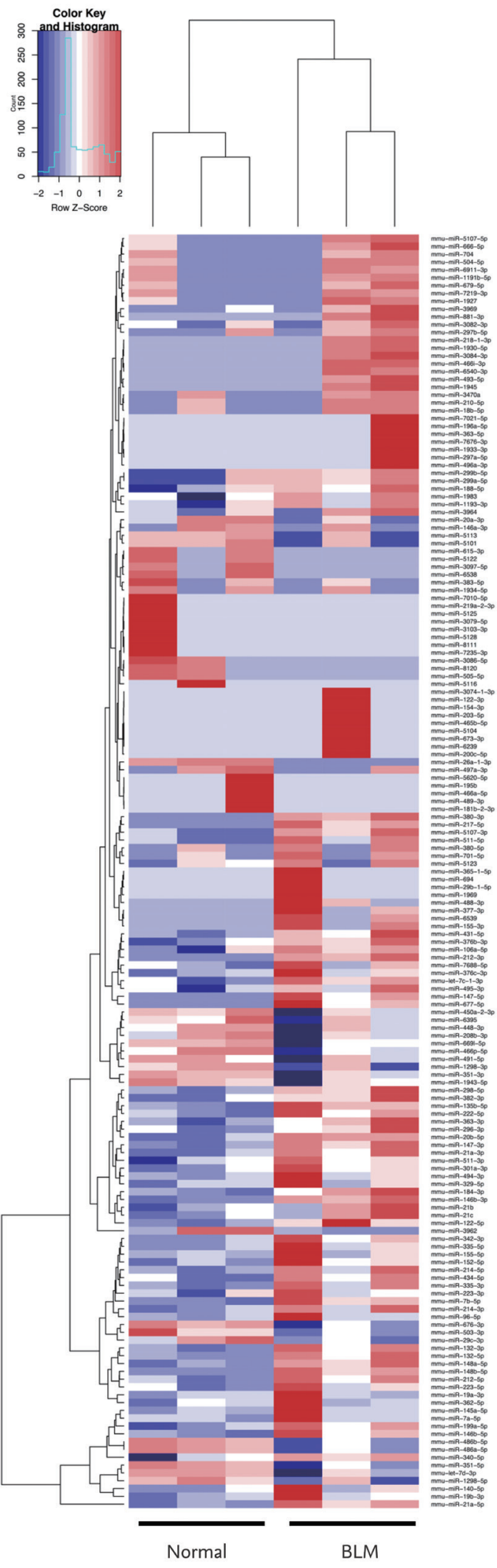

Supplementary Figure 2. Clusters of differentially expressed microRNAs (miRNAs) in bleomycin (BLM)-induced lung fibrosis mice comparing with normal ones. There were 621 miRNAs expressed above background. Each row of the heat map represented one of the differentially expressed miRNA gene, and each column represented a sample. Red indicated an increase in miRNA gene expression, whereas blue indicated a decrease. Samples were identified at the bottom. miRNA names were listed to the right. 